Case Report

\title{
Concurrent Central Diabetes Insipidus and Acute Myeloid Leukemia
}

\author{
Stephanie L. Pritzl ${ }^{D}$, Daniel R. Matson $\left(\mathbb{D}\right.$, Mark B. Juckett ${ }^{D}$, and David J. Ciske \\ University of Wisconsin School of Medicine and Public Health, Madison, Wisconsin, USA \\ Correspondence should be addressed to David J. Ciske; dciske@dshealthcare.com
}

Received 18 September 2020; Revised 2 February 2021; Accepted 8 February 2021; Published 16 February 2021

Academic Editor: Håkon Reikvam

Copyright (C 2021 Stephanie L. Pritzl et al. This is an open access article distributed under the Creative Commons Attribution License, which permits unrestricted use, distribution, and reproduction in any medium, provided the original work is properly cited.

Central diabetes insipidus (CDI) is a rare reported complication of acute myeloid leukemia (AML). The onset of AML-associated CDI often precedes the diagnosis of AML by weeks or months and is considered an adverse prognostic indicator in this setting. The mechanism of AML-associated CDI is not known; however, it is often reported in the setting of cytogenetic events resulting in MDS1 and EVI1 complex locus protein (MECOM) gene overexpression. Here, we describe a case of new onset CDI which preceded a diagnosis of AML by 1 month. We detail the clinical and laboratory evaluation of the patient's CDI, and we describe the pathological and laboratory workup of their AML, which ultimately yielded a diagnosis of AML with myelodysplasia-related changes. Additional cytogenetic findings included the identification of the $\mathrm{t}(2 ; 3)(\mathrm{p} 23 ; \mathrm{q} 27)$, which leads to MECOM gene overexpression and which to our knowledge has not previously been reported in the setting of AML-associated CDI. The patient received induction chemotherapy followed by allogeneic hematopoietic stem cell transplantation but experienced disease relapse and passed away nine months after initial diagnosis. We emphasize that new onset CDI can occur as a rare complication of AML where it portends a poor prognosis and may be related to AML subtypes displaying MECOM gene dysregulation.

\section{Introduction}

Central diabetes insipidus (CDI) as a complication of acute myeloid leukemia (AML) is rare, and the underlying mechanism(s) of AML-associated CDI remains incompletely understood. When it occurs, the onset of CDI typically precedes the diagnosis of AML by 1-2 months [1]. However, CDI may also occur at the time of AML diagnosis or as the initial manifestation of AML relapse $[1,2]$. AMLassociated CDI is hypothesized to represent an adverse prognostic indicator of AML, even when CDI symptoms are ameliorated by administration of desmopressin (DDAVP) [1-3]. Here, we present a case of CDI preceding an initial diagnosis of AML.

\section{Case Presentation}

A previously healthy 71-year-old man presented to his primary care physician with abrupt onset of polyuria and polydipsia. Physical exam was notable for intact visual fields and no evidence of hypovolemia. His medications included only aspirin ( $81 \mathrm{mg}$ daily) and simvastatin ( $40 \mathrm{mg}$ daily). Laboratory testing revealed leukopenia and macrocytic anemia (Table 1), in the setting of a previously normal complete blood count $(\mathrm{CBC})$ one year prior. Comprehensive metabolic panel, hemoglobin A1c, and urinalysis were unremarkable. However, urine osmolality was inappropriately low in conjunction with an elevated serum osmolality (Table 1). Polyuria was confirmed with a 24 -hour urine collection. An 8-hour water deprivation test was completed and demonstrated worsening hypernatremia with a suboptimal response in urinary concentration (Table 1). A subsequent desmopressin challenge was notable for decreased urine output and increased urine osmolality (Table 1). Levels of plasma vasopressin and copeptin, which comprises a portion of the vasopressin precursor molecule, were not measured. Other hormones dependent on pituitary function including serum prolactin, free T4, cortisol (AM), 
TABLE 1: Laboratory data.

\begin{tabular}{|c|c|c|c|}
\hline \multicolumn{4}{|c|}{ Laboratory testing at diagnosis } \\
\hline Assay & Result & \multicolumn{2}{|c|}{ Normal values } \\
\hline WBC & $2.3 \mathrm{~K} / \mu \mathrm{L}$ & \multicolumn{2}{|c|}{$3.8-10.5 \mathrm{~K} / \mu \mathrm{L}$} \\
\hline $\mathrm{Hb}$ & $13.5 \mathrm{~g} / \mathrm{dL}$ & \multicolumn{2}{|c|}{$13.6-17.2 \mathrm{~g} / \mathrm{dL}$} \\
\hline $\mathrm{MCV}$ & $101 \mathrm{fL}$ & \multicolumn{2}{|c|}{$80-97 \mathrm{fL}$} \\
\hline 24-hour urine collection & $12.5 \mathrm{~L}$ urine output & \multicolumn{2}{|c|}{$0.8-2.0 \mathrm{~L}$} \\
\hline Prolactin & $8.9 \mathrm{ng} / \mathrm{mL}$ & \multicolumn{2}{|c|}{$3.5-19.4 \mathrm{ng} / \mathrm{mL}$} \\
\hline Cortisol (AM) & $18.6 \mu \mathrm{g} / \mathrm{dL}$ & \multicolumn{2}{|c|}{$10-20 \mu \mathrm{g} / \mathrm{dL}$} \\
\hline Free $\mathrm{T} 4$ & $0.81 \mathrm{ng} / \mathrm{L}$ & \multicolumn{2}{|c|}{$0.70-1.48 \mathrm{ng} / \mathrm{L}$} \\
\hline Total testosterone & $338 \mathrm{ng} / \mathrm{dL}$ & \multicolumn{2}{|c|}{$300-720 \mathrm{ng} / \mathrm{dL}$} \\
\hline \multicolumn{4}{|l|}{ 8-hour water deprivation test } \\
\hline Assay & 0 hours & 8 hours & Normal values \\
\hline Serum sodium & $144 \mathrm{mmol} / \mathrm{L}$ & $149 \mathrm{mmol} / \mathrm{L}$ & $136-145 \mathrm{mmol} / \mathrm{L}$ \\
\hline Serum osmolality & $302 \mathrm{mOsm} / \mathrm{kg}$ & $308 \mathrm{mOsm} / \mathrm{kg}$ & $278-298 \mathrm{mOsm} / \mathrm{kg}$ \\
\hline Urine osmolality & $123 \mathrm{mOsm} / \mathrm{kg}$ & $235 \mathrm{mOsm} / \mathrm{kg}$ & $50-1200 \mathrm{mOsm} / \mathrm{kg}$ \\
\hline \multicolumn{4}{|l|}{ Desmopressin challenge } \\
\hline Time & Urine output (mL) & \multicolumn{2}{|c|}{ Urine osmolality (mOsm/kg) } \\
\hline 12-1 PM & 725 & \multicolumn{2}{|l|}{ 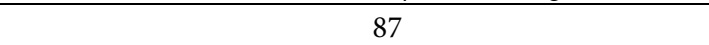 } \\
\hline $1 \mathrm{PM}$ & \multicolumn{3}{|c|}{2 mcg DDAVP SubQ } \\
\hline $1-2 \mathrm{PM}$ & 160 & \multicolumn{2}{|l|}{ 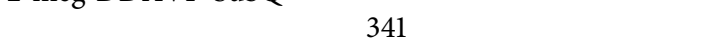 } \\
\hline $2-3 \mathrm{PM}$ & 80 & \multicolumn{2}{|c|}{467} \\
\hline
\end{tabular}

and free testosterone were all within normal limits. MRI head demonstrated lack of the normal posterior pituitary bright spot. The laboratory and imaging findings were consistent with CDI, and the, patient began treatment with DDAVP nasal spray which led to rapid improvement in his symptoms.

During this 21-day period, serial CBCs revealed a persistent leukopenia (WBC $3.4 \mathrm{~K} / \mu \mathrm{L}$ ), worsening macrocytic anemia ( $\mathrm{Hb} 11.7 \mathrm{~g} / \mathrm{Dl}$ and $\mathrm{MCV} 107 \mathrm{fL})$, and a progressive thrombocytosis (PLT $500 \mathrm{~K} / \mu \mathrm{L}$ and normal $160-370 \mathrm{~K} / \mu \mathrm{L}$ ). A manual differential performed on peripheral blood revealed $17 \%$ circulating blasts, and bone marrow biopsy demonstrated a hypercellular marrow virtually effaced by leukemic blasts (Figure 1(a)). Flow cytometry revealed a myeloid immunophenotype confirming a diagnosis of AML, without features worrisome for acute promyelocytic leukemia. Routine ancillary testing for newly diagnosed AML includes (minimally) the following studies performed on the leukemic blast population: karyotyping by classical cytogenetics, evaluation for core binding factor rearrangements by either fluorescence in situ hybridization (FISH) or classical cytogenetics, and mutational analysis of the fms-like tyrosine kinase 3 (FLT3) gene [4].

In this case, cytogenetic analyses revealed the $t(2 ; 3)(\mathrm{p} 23$; q27), which is a chromosomal translocation often associated with MDS1 and EVI1 complex locus (MECOM) gene overexpression, as well as the del (5) q31;q33) (Figure 1(b)). FISH was negative for core binding factor rearrangements, and molecular analyses revealed no mutation in FLT3. Based on the presence of the del (5)(q31;q33), a final diagnosis of AML with myelodysplasia-related changes (AML-MRC) was rendered. AML-MRCs are a subtype of AML that are associated with a poor prognosis. They are diagnosed when AML patients have a known history of myelodysplastic syndrome, display significant morphologic dysplasia in the bone marrow at leukemic presentation, or when their AML harbors characteristic cytogenetic findings that are associated with myelodysplasia [5]. These characteristic cytogenetic findings include deletions in chromosome 5, as seen in our patient.

The patient received induction chemotherapy with liposomal daunorubicin and cytarabine which failed to reduce his bone marrow blast count. This was followed by two cycles of azacytidine and lenalidomide and reinduction with fludarabine, cytarabine, and granulocyte colony-stimulating factor. Three weeks following his second induction, the bone marrow blast count percentage was $95 \%$. He then received an allogeneic stem cell transplantation (alloSCT) following pretreatment with an experimental radiolabeled anti-CD45 monoclonal antibody (IOMAB-01). Unfortunately, he experienced disease relapse 1 month after transplant and ultimately passed away 9 months after his initial diagnosis. Interestingly, prior to transplant, he required $40 \mathrm{mcg} /$ day of DDAVP $0.01 \%$ nasal spray ( $20 \mathrm{mcg}$ twice daily) to control his CDI symptoms, but following his transplant, he was able to space out the dosing of his DDAVP to every 4-5 days.

\section{Discussion}

AML-associated CDI is a rare but increasingly recognized complication of AML. In perhaps the largest study of AMLassociated CDI to date, Ladigan et al. analyzed 51 reports of adults with myeloid malignancies and associated CDI [1]. They found a median age of diagnosis of 48 years, which is younger than the median age of 65 years reported across all new AML diagnoses [6]. At least 9 cases have also been reported in the pediatric population [7-13]. In addition, 


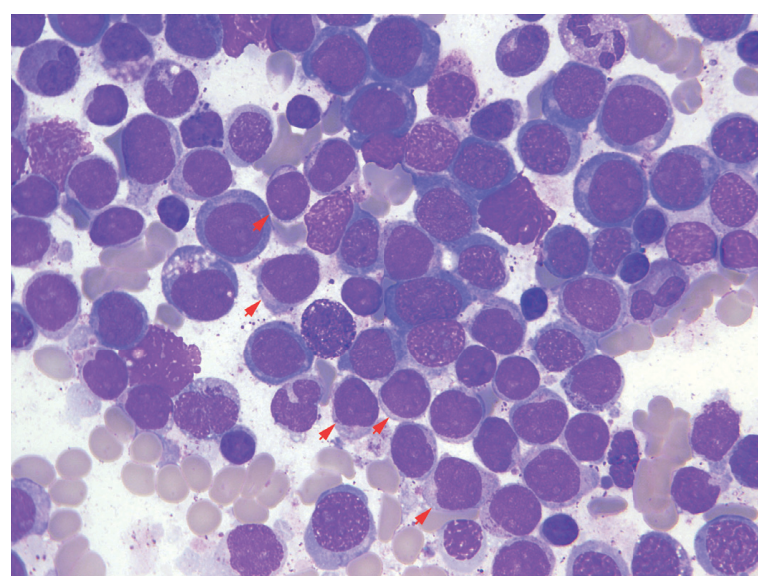

(a)

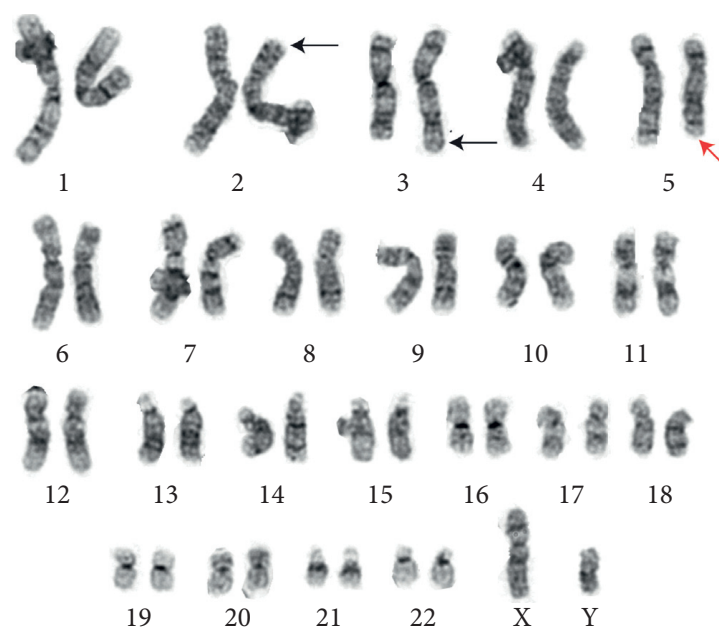

(b)

FIgURE 1: Bone marrow aspirate and AML cytogenetic karyotype: (a) wright-stained bone marrow aspirate showing increased blasts (examples highlighted by red arrowhead; 1000x original magnification); (b) AML karyotype demonstrating the t (2;3)(p23;q27) (black arrows) and del (5q)(q31;q33) (red arrow).

patients with AML-associated CDI show a female predominance (59\% female), compared to a mild male predominance across all AMLs. The majority (45/51) of these cases occur in the setting of de novo AMLs, wherein the patient has no known prior diagnosis of a primary bone marrow neoplasm. The remaining cases are comprised of either myelodysplastic syndrome (MDS), which is a common precursor to AML, or AML transformed from aplastic anemia, MDS, or chronic myelomonocytic leukemia (CMML).

The diagnosis of AML-associated CDI is essentially identical to the diagnosis of CDI in any other setting $[14,15]$. Most patients (75\%) will be diagnosed with CDI concurrently with their AML diagnosis, with most remaining cases presenting no more than 2 months prior to or following their AML diagnosis [1]. While the majority of patients with AML-associated CDI (61\%) will have no associated brain imaging abnormalities, some may have pathologic findings including loss of the posterior pituitary bright spot or nodular thickening/attenuation of the pituitary stalk [1]. MRI of our patient revealed absence of the posterior pituitary bright spot which can be indicative of hypothalamic pituitary dysfunction. The normal pituitary bright spot seen on T1-weighted MRI is thought to result from the T1shortening effect of stored vasopressin in the posterior pituitary [16].

The pathogenesis of AML-associated CDI remains unclear although there are hints that dysmegakaryopoiesis and resulting platelet dysfunction could lie at the heart of this disorder. Previously, clinicians hypothesized that CDI occurred secondary to pituitary damage as a consequence of tumor-mediated destruction or infection. Early cohort studies of deceased patients with leukemia-associated CDI noted two types of lesions that could be routinely identified at autopsy in the supraopticohypophyseal system: (1) leukemic infiltrates and/or (2) thrombosis of small vessels [16].
However, it should be noted that leukemic infiltrates are not uncommonly seen at autopsy in AML patients who never developed CDI $[2,17,18]$. More recently, multiple case series have revealed a strong association between AMLassociated CDI and cytogenetic abnormalities in the concurrent AML, chiefly inversions involving chromosome 3 (inv (3)) and monosomies involving chromosome 7 (monosomy 7) [1, 3, 19, 20]. Interestingly, at least a subset of cases with inv (3) harbor fusion events leading to MECOM gene overexpression, which has been shown to drive both leukemogenesis and dysmegakaryopoiesis [3, 21, 22]. It is theorized that the resulting abnormal platelets could adversely impact circulating vasopressin function, as $90 \%$ of peripheral vasopressin is platelet-bound [1-3]. Cytogenetic studies in our patient revealed the $t(2 ; 3)(\mathrm{p} 23 ; \mathrm{q} 27)$, a translocation that also typically results in MECOM overexpression and which to our knowledge has not been previously reported in the setting of AML-associated CDI [23]. Interestingly, our patient also showed a progressive thrombocytosis, an uncommon event in AML that has nonetheless been reported in the setting of the $t(2 ; 3)$ and MECOM overexpression [24]. Finally, our patient showed a greatly decreased requirement for DDAVP following alloSCT, after which his circulating platelets would have been derived from donor marrow. This decreased requirement for DDAVP and even complete resolution of CDI following AML treatment has been previously reported [1] and argues against pituitary destruction as the cause of AML-associated CDI in these cases.

Clinicians treating a patient with AML-associated CDI must correct the patient's CDI while also identifying an optimal treatment strategy for their AML. 90\% of reported AML-associated CDI cases have a documented response to a vasopressin analog (mainly DDAVP), with the majority of patients demonstrating a marked improvement in CDI symptoms upon treatment [1]. Our patient's CDI symptoms 
are rapidly improved with DDAVP nasal spray. In comparison, treatment of the underlying AML depends on a number of factors, including the presence or absence of prognostically important cytogenetic and molecular findings and the patient's ability to tolerate therapy. In this respect, it is important to note that both monosomy 7 and inv (3) constitute unfavorable-risk cytogenetic findings in AML and are independently associated with worse rates of complete remission and overall survival [25-27]. Thus, independent of CDI, most AML-associated CDI patients would already be expected to have a 5-year AML-related survival of only 5$10 \%$, compared to a 5-year survival of $29 \%$ across all AMLs [6]. In general, there is also a paucity of data regarding optimal induction regimens for AML patients with associated CDI. In 39 patients who received treatment with either standard-dose cytarabine and daunorubicin (often referred to as $7+3$ ) or high-dose cytarabine, the rate of complete remission (CR) was only 15\% [28-33]. Sufficient reports of other treatment regimens are not readily available. Perhaps not surprisingly given these data, AMLs in patients with AML-associated CDI confer a dismal prognosis, with an overall 1-year survival as low as $20 \%$ and a median overall survival time of only 6 months $[1,3]$. However, CDI-associated AML patients who underwent alloSCT showed markedly better outcomes, with $40 \%$ surviving to 12 months. This is consistent with the survival benefit seen with alloSCT following first $\mathrm{CR}$ in $\mathrm{AML}$ patients with unfavorable risk cytogenetics [34]. Thus, the best available evidence suggests that patients with AML-associated CDI typically have unfavorable-risk cytogenetics and stand to benefit from intensive induction chemotherapy regimens followed (when possible) by an alloSCT in the first CR. Unfortunately, our patient never achieved a CR despite receiving multiple induction regimens, and although he underwent an alloSCT facilitated by pretreatment with an experimental therapy, he rapidly relapsed.

We reiterate that CDI is a rare but recognized complication of AML which is associated with a poor prognosis. We support future studies examining the consequences of MECOM gene dysregulation in AML and vasopressin function in both the pituitary and peripheral circulation.

\section{Data Availability}

No data sets were generated in the preparation of this report.

\section{Disclosure}

This case report was prepared as part of the employment of the authors at the University of Wisconsin Hospitals and Clinics.

\section{Conflicts of Interest}

The authors declare that there are no conflicts of interest.

\section{Acknowledgments}

The authors would like to acknowledge the Wisconsin State Lab of Hygiene for providing images of the cytogenetic findings in this case. DRM is further supported by Grant number T32-HL007899 from the NHLBI.

\section{References}

[1] S. Ladigan, T. Mika, A. Figge et al., "Acute myeloid leukemia with central diabetes insipidus," Blood Cells, Molecules, and Diseases, vol. 76, pp. 45-52, 2019.

[2] P. Dy, P. Chua, J. Kelly, and S. Liebman, "Central diabetes insipidus in the setting of acute myelogenous leukemia," American Journal of Kidney Diseases, vol. 60, no. 6, pp. 998-1001, 2012.

[3] E. H. Cull, J. M. Watts, M. S. Tallman et al., "Acute myeloid leukemia presenting with panhypopituitarism or diabetes insipidus: a case series with molecular genetic analysis and review of the literature," Leukemia \& Lymphoma, vol. 55, no. 9, pp. 2125-2129, 2014.

[4] D. A. Arber, M. J. Borowitz, M. Cessna et al., "Initial diagnostic workup of acute leukemia: guideline from the college of American pathologists and the American society of hematology," Archives of Pathology \& Laboratory Medicine, vol. 141, no. 10, pp. 1342-1393, 2017.

[5] D. A. Arber and H. P. Erba, "Diagnosis and treatment of patients with acute myeloid leukemia with myelodysplasiarelated changes (AML-MRC)," American Journal of Clinical Pathology, vol. 154, no. 6, pp. 731-741, 2020.

[6] NIH, Acute Myeloid Leukemia-Cancer Stat Facts, NIH, Bethesda, MD, USA, 2018, https://seer.cancer.gov/statfacts/ html/amyl.html.

[7] M. C. Joseph and S. E. Levin, "Leukaemia and diabetes insipidus," BMJ, vol. 1, no. 4979, pp. 1328-1322, 1956.

[8] G. E. Bergman, H. J. Baluarte, and J. L. Naiman, "Diabetes insipidus as a presenting manifestation of acute myelogenous leukemia," The Journal of Pediatrics, vol. 88, no. 2, p. 355, 1976.

[9] D. J. Kanabar, D. R. Betts, B. Gibbons, J. E. Kingston, and O. B. Eden, "Monosomy 7, diabetes insipidus and acute myeloid leukemia in childhood," Pediatric Hematology and Oncology, vol. 11, no. 1, pp. 111-114, 1994.

[10] S. Roy and W. W. Johnson, "Diabetes insipidus in a child with erythromyelocytic leukemia," Archives of Pediatrics \& Adolescent Medicine, vol. 119, no. 1, pp. 82-85, 1970.

[11] U. Betkerur, A. Shende, and P. Lanzkowsky, "Acute myeloblastic leukemia presenting with diabetes insipidus," The American Journal of the Medical Sciences, vol. 273, no. 3, pp. 325-328, 1977.

[12] H. A. Frangoul, D. W. W. Shaw, D. Hawkins, and J. Park, "Diabetes insipidus as a presenting symptom of acute myelogenous leukemia," Journal of Pediatric Hematology/Oncology, vol. 22, no. 5, pp. 457-459, 2000.

[13] W. Wössmann, A. Borkhardt, R. Gossen, F.-J. Göbel, and A. Reiter, "Acute myeloid leukemia presenting with diabetes insipidus," European Journal of Pediatrics, vol. 161, no. 3, pp. 161-162, 2002.

[14] A. Garrahy, C. Moran, and C. J. Thompson, "Diagnosis and management of central diabetes insipidus in adults," Clinical Endocrinology, vol. 90, no. 1, pp. 23-30, 2019.

[15] W. Fenske and B. Allolio, "Current state and future perspectives in the diagnosis of diabetes insipidus: a clinical review," The Journal of Clinical Endocrinology \& Metabolism, vol. 97, no. 10, pp. 3426-3437, 2012.

[16] V. I. Miller and W. G. Campbell, "Diabetes insipidus as a complication of leukemia: a case report with a literature review," Cancer, vol. 28, no. 3, pp. 666-673, 1971. 
[17] K. Yamagami and K. Yoshioka, "Central diabetes insipidus presenting as an early sign of acute myeloid leukemia," Clinical Advances in Hematology \& Oncology: H↔O, vol. 10, no. 6, pp. 400-401, 2012.

[18] K. Loukidis, E. Papadakis, N. Anagnostou et al., "Polyuria due to central diabetes insipidus presenting as an early manifestation of acute myeloid leukemia," Clinical Advances in Hematology \& Oncology: H\&O, vol. 10, no. 6, pp. 399-400, 2012.

[19] C. Castagnola, M. Morra, B. Bemasconi, C. Astori, S. Santagostino, and C. Bemasconi, "Acute myeloid leukemia and diabetes insipidus: results in 5 patients," Acta Haematologica, vol. 93, no. 1, pp. 1-4, 1995.

[20] A. De La Chapelle and R. Lahtinen, "Monosomy 7 predisposes to diabetes insipidus in leukaemia and myelodysplastic syndrome," European Journal of Haematology, vol. 39, no. 5, pp. 404-411, 1987.

[21] M. Sonmez, N. Erkut, T. S. Tat, F. Celep, U. Cobanoglu, and H. O. Ersoz, "Can a high platelet count be responsible for diabetes insipidus in acute myelogenous leukemia with monosomy 7 and inversion 3 (q21q26)?" International Journal of Hematology, vol. 90, no. 2, pp. 273-274, 2009.

[22] K. Morishita, E. Parganas, C. L. William et al., "Activation of EVI1 gene expression in human acute myelogenous leukemias by translocations spanning 300-400 kilobases on chromosome band 3q26," Proceedings of the National Academy of Sciences, vol. 89, no. 9, pp. 3937-3941, 1992.

[23] J. L. Huret, M. Ahmad, M Arsaban et al., "Atlas of genetics and cytogenetics in oncology and haematology in 2013," Nucleic Acids Research, vol. 41, no. Database issue, pp. D920-D924, 2013.

[24] K. Yamamoto, A. Okamura, Y. Sanada, K. Yakushijin, H. Matsuoka, and H. Minami, "Marked thrombocytosis and dysmegakaryopoiesis in acute myeloid leukemia with $\mathrm{t}(2 ; 3)$ (p22; q26.2) and EVI1 rearrangement," Annals of Hematology, vol. 92, no. 12, pp. 1713-1715, 2013.

[25] D. Grimwade, H. Walker, F. Oliver et al., "The importance of diagnostic cytogenetics on outcome in AML: analysis of 1,612 patients entered into the MRC AML 10 trial," Blood, vol. 92, no. 7, pp. 2322-2333, 1998.

[26] M. L. Slovak, K. J. Kopecky, P. A. Cassileth et al., "Karyotypic analysis predicts outcome of preremission and postremission therapy in adult acute myeloid leukemia: a Southwest oncology group/eastern cooperative oncology group study," Blood, vol. 96, no. 13, pp. 4075-4083, 2000.

[27] J. C. Byrd, K. Mrózek, R. K. Dodge et al., "Pretreatment cytogenetic abnormalities are predictive of induction success, cumulative incidence of relapse, and overall survival in adult patients with de novo acute myeloid leukemia: results from cancer and leukemia group B (CALGB 8461)," Blood, vol. 100, no. 13, pp. 4325-4336, 2002.

[28] F. Nakamura, Y. Kishimoto, T. Handa, Y. Arai, and K. Mitani, "Myelodysplastic syndrome with central diabetes insipidus manifesting hypodipsic hypernatremia and dehydration," American Journal of Hematology, vol. 75, no. 4, pp. 213-216, 2004.

[29] R. P. Mozersky, V. K. Bahl, D. Meisner, and H. Patel, "Diabetes insipidus, acute myelogenous leukemia, and monosomy 7," The Journal of the American Osteopathic Association, vol. 96, no. 2, pp. 116-118, 1996.

[30] P. Nieboer, E. Vellenga, R. Adriaanse, and A. A. van de Loosdrecht, "Central diabetes insipidus preceding acute myeloid leukemia with $\mathrm{t}(3 ; 12)$ (q26;p12)," The
Netherlands Journal of Medicine, vol. 56, no. 2, pp. 45-47, 2000.

[31] L. Pagano, M. T. Voso, S. Sica, and G. Leone, "Recovery from diabetes insipidus associated with AML after a BMT conditioning regimen including busulfan," Bone Marrow Transplantation, vol. 11, no. 2, pp. 175-176, 1993.

[32] C. C. Yen, C. H. Tzeng, J. H. Liu et al., "Acute myelomonocytic leukemia preceded by secondary amenorrhea and presenting with central diabetes insipidus: a case report," Zhonghua Yi Xue Za Zhi, vol. 60, no. 4, pp. 213-218, 1997.

[33] A. Lawrie, D. A. J. Stevenson, T. N. Doig, M. A. Vickers, and D. J. Culligan, "Acute myeloid leukemia presenting in a mother and daughter pair with the identical acquired karyotypic abnormality consisting of inversion 3q21q26 and monosomy 7: a review of possible mechanisms," Cancer Genetics, vol. 205, no. 11, pp. 599-602, 2012.

[34] W.-J. Hong and B. C. Medeiros, "Unfavorable-risk cytogenetics in acute myeloid leukemia," Expert Review of Hematology, vol. 4, no. 2, pp. 173-184, 2011. 